\title{
Visual perception of the relative phasing of human limb movements
}

\author{
GEOFFREY P. BINGHAM \\ Indiana University, Blooomington, Indiana \\ RICHARD C. SCHMIDT \\ College of the Holy Cross, Worcester, Massachusetts \\ and \\ FRANK T. J. M. ZAAL \\ Indiana University, Bloomington, Indiana
}

\begin{abstract}
Studies of bimanual coordination have found that only two stable relative phases $\left(0^{\circ}\right.$ and $\left.180^{\circ}\right)$ are produced when a participant rhythmically moves two joints in different limbs at the same frequency. Increasing the frequency of oscillation causes an increase in relative phase variability in both of these phase modes. However, relative phasing at $180^{\circ}$ is more variable than relative phasing at $0^{\circ}$, and when the frequency of oscillation reaches a critical frequency, a transition to $0^{\circ}$ occurs. These results have been replicated when 2 people have coordinated their respective limb movements using vision. This inspired us to investigate the visual perception of relative phase. In Experiment 1, recordings of human interlimb oscillations exhibiting different frequencies, mean relative phases, and different amounts of phase variability were used to generate computer displays of spheres oscillating either side to side in a frontoparallel plane or in depth. Participants judged the stability of relative phase. Judgments covaried with phase variability only when the mean phase was $0^{\circ}$ or $180^{\circ}$. Otherwise, judgments covaried with mean relative phase, even after extensive instruction and demonstration. In Experiment 2, mean relative phase and phase variability were manipulated independently via simulations, and participants were trained to perceive phase variability in testing sessions in which mean phase was held constant. The results of Experiment 1 were replicated. The HKB model was fitted to mean judgment standard deviations.
\end{abstract}

Phase refers to the proportion of the cycle traveled at a given time in a rhythmic motion. If the motion is represented as a trajectory on the phase plane (i.e., a plot of velocity vs. position), then the phase is the angular coordinate of the motion (measured in degrees or radians). The relative phase of two motions (e.g., the swinging of two legs) is the difference of the two phases. A number of studies have shown that, when a person oscillates two equivalent limbs at a common frequency and each about a single joint, then one of only two stable relative phase relations is exhibited, either $0^{\circ}$ or $180^{\circ}$ relative phase (Kelso, 1984, 1995; Kelso, Schoner, Scholz, \& Haken, 1987; Scholz, Kelso, \& Schoner, 1987; Schoner \& Kelso, 1988; Tuller \& Kelso, 1989; Yaminishi, Kawato, \& Suzuki, 1979, 1980). For instance, Tuller and Kelso (1989) used two metronomes

Portions of this work were presented at the annual meeting of the Psychonomic Society, November 1996 (Bingham, Schmidt, \& Zaal, 1996; Zaal, Bingham, \& Schmidt, 1996). The authors wish to acknowledge the assistance of Michael Stevens in the data collection of Experiment 1, Michael Stassen in programming the simulations of Experiment 2, and James Craig. Tim Lee, and an anonymous reviewer for valuable suggestions about methodology and analysis. Correspondence to should be addressed to G. P. Bingham, Department of Psychology, Indiana University, Bloomington, IN 47405 (e-mail: gbingham@indiana.edu). to help participants to try to oscillate their left and right index fingers at relative phases other than $0^{\circ}$ and $180^{\circ}$. Participants coordinated each finger with one of the two metronomes. They succeeded only in stably oscillating at required relative phases of $0^{\circ}$ and $180^{\circ}$ (i.e., at an in-phase or antiphase relation). Otherwise, their mean relative phase tended to deviate from that required, drifting toward the closer of the two stable phases. Also the standard deviation $(S D)$ of relative phase was higher at phases other than $0^{\circ}$ and $180^{\circ}$.

Furthermore, Kelso (1984) has shown that a person oscillating at $180^{\circ}$ exhibits enough of an increase in the $S D$ of relative phase with increasing frequency of oscillation that a switch to $0^{\circ}$ relative phase occurs, and, at that point, the $S D$ drops to the level maintained by a $0^{\circ}$ relative phase oscillation. A person oscillating at $0^{\circ}$ will not switch as frequencies are increased.

This switching behavior inspired Haken, Kelso, and Bunz (1985) to develop the $H K B$ model of bimanual coordination. As shown in Figure 1, the model is in the form of a potential function, $V(\phi)$, which represents the relative amount of energy required to maintain coordinated oscillation at a given relative phase. Haken et al. used a phenomenological approach and wrote the potential function with minima at $0^{\circ}$ and $180^{\circ}$ to fit the observed stabilities. 
HKB model: $V(\phi)=-a \cos (\phi)-b \cos (2 \phi)$

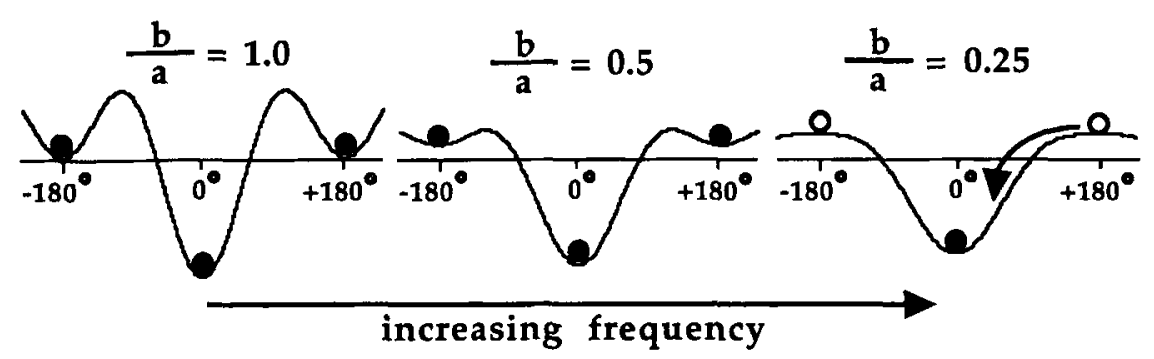

Figure 1. Illustration of the HKB model showing the form of the potential function and its dependence on the ratio of the parameters. The ratio is used to capture changes in behavior that occur with increasing frequency of oscillation. See the text for additional explanation. After Kelso (1995).

The relative values of two parameters, $a$ and $b$, determine the exact form of the potential function and are used to model the effect of increasing frequency of oscillation. As the ratio $b / a$ goes below .25 , the minima at $180^{\circ}$ phase is eliminated, rendering the $180^{\circ}$ phase unstable. The dynamical model is written using relative phase (i.e., $\phi$ ) as a state variable rather than the positions and/or velocities of the oscillating limbs. Haken et al. refer to $\phi$ as an order parameter and describe it as a macroscopic variable that captures the organization in the behavior under study. Given the relative success of the model, $\phi$ would indeed seem to be descriptively efficacious. Nevertheless, it remains unclear what role $\phi$ plays in the organization and control of the behavior. Might relative phase be perceived and controlled? In the original experiments (e.g., Kelso, 1984), participants were told to perform oscillations at a given relative phase (e.g., $0^{\circ}$ or $180^{\circ}$ ). This implies that relative phase is a property of coordinated movements that participants can perceive, at least, well enough to know that they are fulfilling the requirements of the task.

The supposition that relative phase is a perceptible property is supported by results of a study in which the two oscillating limbs were those of different people. Schmidt, Carello, and Turvey (1990) asked 2 participants to oscillate equivalent limbs in either $0^{\circ}$ or $180^{\circ}$ relative phase as the frequency of oscillation was increased. This study reproduced the interlimb behaviors found in previous studies (e.g., increasing phase variability before switching), but this time with the relation between the limbs mediated by vision. In this case, relative phase must have been a visible property. On the other hand, participants have sometimes been unaware that relative phase has deviated from that which they intend to produce. Because the limbs being coordinated in the Schmidt et al. (1990) study were those of 2 different people, they differed in their inertia and, consequently, their inherent frequency of oscillation. This difference in inherent frequency of oscillation, captured as $\Delta \omega\left(=\omega_{1}-\omega_{2}\right)$ in a revised HKB model (Fuchs \& Kelso, 1994; Kelso \& Jeka,
1992; Schmidt \& Turvey, 1995), requires that both oscillators must move from their inherent frequency to oscillate isochronously. This produces what is called fixedpoint drift - the mean relative phase deviates from either $0^{\circ}$ or $180^{\circ}$ as the frequency of oscillation is increased. Participants seemed to be unaware of this drift. Numerous other studies (e.g., Bingham, Schmidt, Turvey, \& Rosenblum, 1991; Kelso \& Jeka, 1992; Rosenblum \& Turvey, 1988; Schmidt, Shaw, \& Turvey, 1993; Sternad, Turvey, \& Schmidt, 1992; Turvey, Rosenblum, Schmidt, \& Kugler, 1986) have replicated this effect by explicitly manipulating $\Delta \omega$ through alterations of the inertial properties of the two limbs. These studies have established that $\Delta \omega$ is another control parameter that manipulates the dynamics of relative phase. In particular, these studies have shown that, as the $\Delta \omega$ deviates from 0 (i.e., identical inertial loadings), both the deviations from intended relative phase $\left(0^{\circ}\right.$ and $180^{\circ}$ ) and relative phase variability increase.

While relative phase has been manipulated in past studies investigating visual event recognition (e.g., Bertenthal \& Pinto, 1993; Johansson, 1950/1994), the visual perception of relative phase as such has not been investigated directly. The questions addressed by the present study were motivated by results from human movement studies. If people can use vision to maintain selected relative phases of limb movement, does this mean that people can perceive relative phase, and, if so, how well? The most convenient method by which to address this question would have been to have participants make judgments of simulated oscillators in which the relative phase and phase variability are manipulated. However, the variability of relative phase in bimanual movements has been found to be uniquely structured. Spectral analysis has revealed that the power of the relative phase spectrum increases as $1 / f$, that peaks occur at integer multiples of the oscillation frequency, and that these peaks decrease with increasing frequency (Schmidt, Beek, Treffner, \& Turvey, 1991). Because it was unclear how to produce displays using simulations that exhibit such properties, we began our investigation of 
the visual perception of relative phase by using recorded kinematics of bimanual rhythmic movements to create visual displays.

We used the recorded motions of two representative participants (Participants 4 and 7) in an experiment reported by Schmidt et al. (1993). In this experiment, participants oscillated two hand-held pendulums at one of two intended phase relations, either $0^{\circ}$ or $180^{\circ}$. As shown in Figure 2A, the pendulums were wooden rods with metal weights attached to the bottom ends and were oscillated by holding the top of each rod firmly in a hand, which rotated about the wrist with the forearm held level. As shown in Figure 2B, three different pendulum lengths were used, each by itself would yield its own inherent frequency of oscillation determined by the simple equivalent pendulum length. Pendulums of the three lengths were combined in pairs to form five wrist-pendulum systems and, consequently, five $\Delta \omega \mathrm{s}$ as shown in Figure $2 \mathrm{C}$. Relative phases other than $0^{\circ}$ and $180^{\circ}$ were generated when participants oscillated together pendulums of unequal lengths. As al- ready described, this manipulation causes $\Delta \omega$-scaled deviations from intended phases of $0^{\circ}$ and $180^{\circ}$ (Figure $3 \mathrm{~A}$ ) as well as $\Delta \omega$-scaled increases in relative phase variability (Figure $3 \mathrm{C}$ ). Because both relative phase variability and relative phase deviation are scaled by $\Delta \omega$, the variability and the deviation are positively correlated $\left[r^{2}(28)\right.$ $=.27, p<.01]$. Participants also oscillated each wristpendulum system at each of three frequencies $(0.80,0.94$, and $1.15 \mathrm{~Hz}$ ), which yielded frequency-scaled deviations from intended phases of $0^{\circ}$ and $180^{\circ}$ (Figure 3B) as well as frequency-scaled increases in relative phase variability (Figure 3D). In all, the experiment had 2 intended relative phases, 5 pendulum systems, and 3 frequencies of oscillation for a total of 30 conditions.

\section{EXPERIMENT 1}

Using displays generated via these recorded bimanual rhythmic movements, we asked participants to judge how well the movements were coordinated-that is, how stable
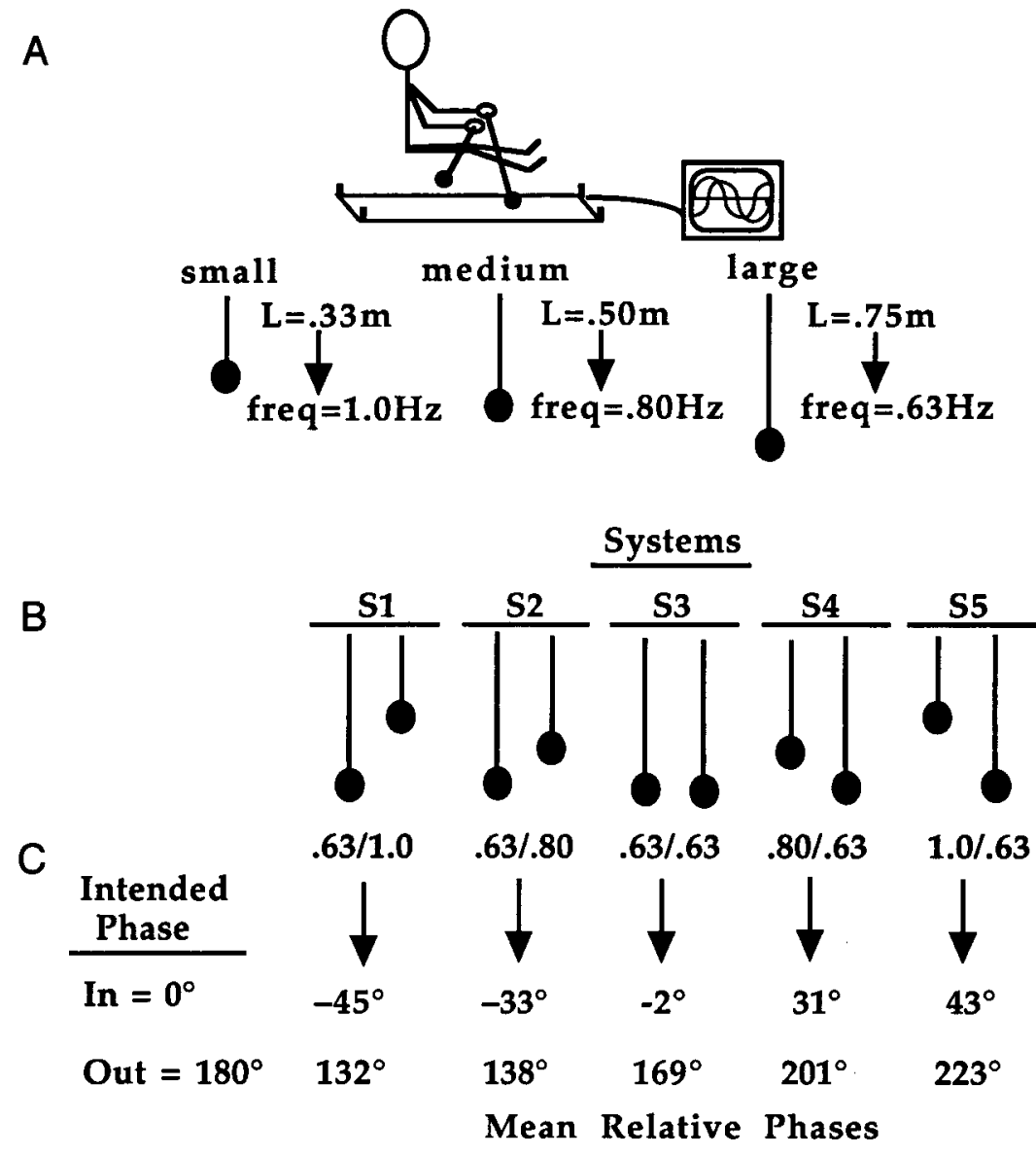

Figure 2. (A) Illustration of the experimental arrangement reported in Schmidt et al. (1993). The participant's legs were raised on a supporting chair so that the sonic digitizer could record the motions of the ends of the wrist pendulums below the participant. (B) Simple equivalent pendulum lengths and frequencies. (C) The configurations of the five wrist-pendulum systems, including the pendulum frequencies and the resulting mean relative phases for each of the two intended phases. 

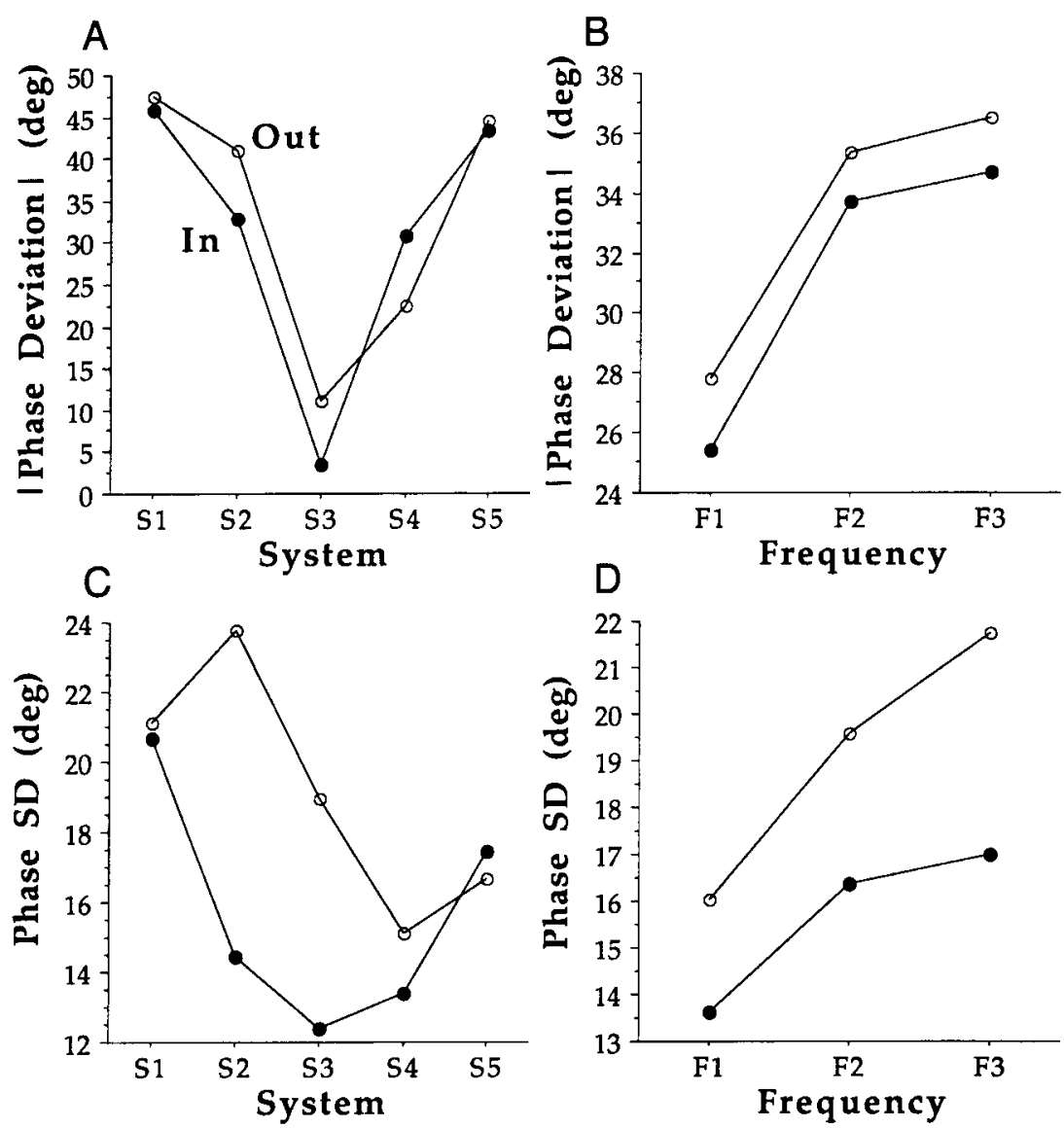

Figure 3. (A) The mean absolute phase deviations that resulted for each of the five wrist-pendulum systems oscillated either at an in-phase (filled circles) or an antiphase (open circles) relation. (B) The mean absolute phase deviations that resulted at each of the three frequencies when wrist pendulums were oscillated either at an in-phase (filled circles) or an anitphase (open circles) relation. $F 1=0.799 \mathrm{~Hz} ; F 2=0.936 \mathrm{~Hz}$; and F3 $=$ $1.151 \mathrm{~Hz}$. (C) The mean standard deviations of relative phase that resulted for each of the five wrist-pendulum systems oscillated either at an in-phase (filled circles) or an anitphase (open circles) relation. (D) The mean standard deviations of relative phase that resulted at each of the three frequencies when wrist pendulums were oscillated either at an in-phase (filled circles) or an anitphase (open circles) relation. $F 1=0.799 \mathrm{~Hz} ; \mathrm{F} 2=$ $0.936 \mathrm{~Hz} ; \mathrm{F3}=1.151 \mathrm{~Hz}$.

the relative phasing of the movement was. One group of participants observed movements in the frontoparallel plane, whereas another group observed movements occurring in the sagittal plane. Two circles oscillated on a computer screen either side to side or in depth (i.e., expanding and contracting) as shown in Figure 4. Participants were asked to judge on an 11 -point scale the level of coordination of the movements defined as the amount of phase variability. A highly coordinated movement with no phase variability received a 10 , and a highly variable poorly coordinated movement received a 0 .

\section{Methods}

Participants. Thirteen undergraduates at Tulane University judged displays of side-to-side movements. Fifteen undergraduates at Indiana University judged displays of movements in depth. All participants received credit in an introductory psychology course for participation. All had normal or corrected-to-normal vision.

Display generation. Displays were programmed on a Macintosh IIci and shown on an Apple 13-in. color display. The program read in the kinematic data from the Schmidt et al. (1993) study and used them to generate the displays. The wrist-pendulum trajectories were sampled at $90 \mathrm{~Hz}$ using an UltraSonic 3-Space Digitizer (Schmidt et al., 1993). These time series were down sampled and used to generate 10-sec displays shown at $33 \mathrm{~Hz}$. Vertical and horizontal coordinates of planar motions of the ends of the pendulums were used Displays of side-to-side motions consisted of two bright dots $(0.2 \mathrm{~cm}$ in diameter) appearing in the center of an otherwise dark screen. The dots represented the ends of the pendulum rods and moved horizontally along curved paths that were separated vertically by a distance of $4 \mathrm{~cm}$ on the screen. Displays of motions in depth consisted of bright rings $(\approx 1 \mathrm{~cm}$ in diameter) appearing in the center of the otherwise dark screen. One ring was $4 \mathrm{~cm}$ above and $4 \mathrm{~cm}$ to the left of the other on the screen. The curvilinear motion of the ends of the 


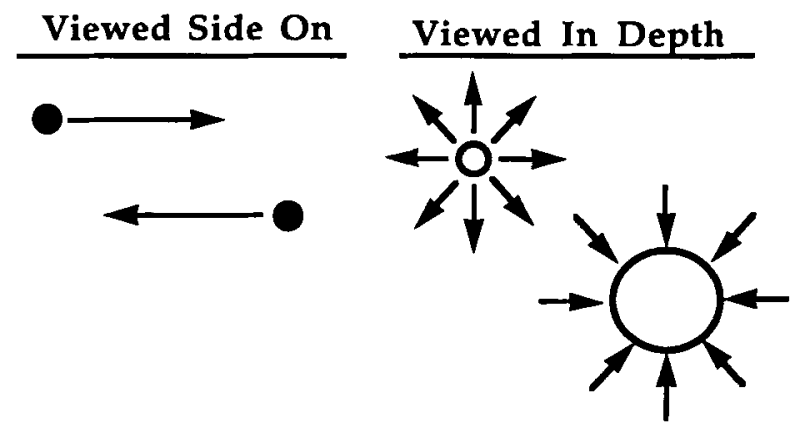

Figure 4. Illustration of the displays for the two viewing conditions.

pendulums in 3-space yielded via scaled orthographic projection (e.g., Nalwa, 1993, p. 39) both expansion/contraction and vertical translation of the rings on the screen. The amplitudes of motion of each of the pendulums in a pair were variable depending on the pendulum system and the participant. Horizontal displacements for side-to-side motions on the screen, for instance, ranged between 3 and $12 \mathrm{~cm}$. Displays were viewed binocularly at a distance of about $1 \mathrm{~m}$ in a dim room.

Procedure. The difference between mean phase and phase variability was explained informally to the participants and illustrated via hand movements showing that a $0^{\circ}$ mean phase could be produced either with low or high phase variability. The remainder of the experiment was automated. The following instructions first appeared on the computer screen:

You will see two white dots moving on the screen. These represent two coordinated rhythmic movements made by people moving their arms. We would like you to judge how coordinated the movements are by making coordination judgments on a scale from 0 to 10 . If the movements are fully coordinated, they would receive a 10 . If they are not coordinated at all they would receive a 0 . After you view a trial, a window will appear in which you move a bar with the mouse to indicate your judgment. In order for you to understand what fully coordinated and not coordinated mean in terms of this task, you will see next three trials. The first two will be fully coordinated, perfect inphase and perfect antiphase coordination, and would receive a coordination judgment score of 10 . The third will be not coordinated at all and would receive a coordination judgment score of 0 . Click on the Done button below to continue.

Next, the participants saw three demonstrations that consisted of harmonic motions, either side to side or in depth as appropriate. The first two were phase locked at $0^{\circ}$ and at $180^{\circ}$ (i.e., with no phase variability). The last demonstration paired motions of unequal frequencies that resulted in phase "winding" (i.e., no stable phase relation). The participants were allowed to view the demonstrations as many times as they wished before proceeding. Finally, the participants viewed and judged experimental trials. A response bar appeared after each display. Below the horizontal bar appeared a scale from 0 to 10 . The participants used the mouse and cursor to move the marker on the bar to the value representing their judgment. They were allowed to adjust the marker freely before double clicking the mouse for the next display. Judgment values along the continuum from 0 to 10 were recorded.

Displays were presented in a completely random order. Five systems $\times 2$ intended phases $\times 3$ frequencies $\times 4$ trials per cell yielded a total of 120 trials that were performed in a session of $1.25 \mathrm{~h}$.

\section{Results}

We computed a mean and an $S D$ of the coordination judgments for each cell and participant. We first performed analyses of variance (ANOVAs) on these values, with intended phase (in phase, antiphase), frequency (1-3), and system (1-5) as within-subjects factors and with viewing condition (side on, in depth) as a between-subjects factor. First, we analyzed the means. Intended phase was not significant $(p>.5)$. System was significant $[F(2,52)=10.8$, $p<.001]$, as were the intended phase $\times \operatorname{system}[F(4,104)=$ $25.5, p<.001]$ and viewing $\times$ intended phase $\times$ system $[F(4,104)=5.4, p<.001]$ interactions. As shown in Figure 5 , the pattern of results was similar whether the viewing was side on or in depth. Mean phases near $0^{\circ}$ and $180^{\circ}$ (i.e., wrist-pendulum system 3 oscillated either in phase or antiphase) were judged as maximally coordinated. As mean relative phase deviated increasingly from $0^{\circ}$ or $180^{\circ}$, the motion was judged as less coordinated. The decrease in judgments was greater with deviations from $0^{\circ}$ than with deviations from $180^{\circ}$. When viewed in depth, $0^{\circ}$ was judged to be more coordinated than $180^{\circ}$, but not when viewed side on. There was a main effect of frequency $[F(2,52)=10.8, p<.001]$, as well as significant frequency $X$ system $[F(8,208)=4.4, p<.001]$ and intended phase $X$ frequency $\times$ system $[F(8,208)=5.4, p<.001]$ interactions. As frequency increased, motions were judged to be less coordinated. This effect was greater for intended inphase motions than for antiphase motions.

When this same ANOVA was performed on judgment $S D$ s, only system $[F(4,104)=4.8, p<.002]$ and the intended phase $\times$ frequency interaction $[F(2,52)=7.0, p<$ $.01]$ were significant. As shown in Figure $6, S D$ s were least for wrist-pendulum system 3 (i.e., for mean relative phases near $0^{\circ}$ and $180^{\circ}$ ). Judgment variability increased as mean phases deviated from either $0^{\circ}$ or $180^{\circ}$.

These analyses revealed that coordination judgments ordered according to the deviations from intended relative phases, decreasing as mean phases either increased or decreased from $0^{\circ}$ or $180^{\circ}$, respectively. However, as stated above, relative phase variability and deviation covary. To test how the combined judgments varied with mean relative phase and phase variability, we performed a multiple regression, regressing absolute phase deviations, phase $S D$ s, and frequencies on mean judgments. We also included independent categorical variables representing intended phase (in phase $=-1$, antiphase $=+1$ ) and viewing condition (side on $=-1$, in depth $=+1$ ) and vectors representing interactions among these factors. We then iteratively removed the factor with the smallest partial $F$ until only significant factors remained (Pedhazur, 1982). The resulting regression accounted for $32 \%$ of the variance $[F(7,832)=55.0, p<.001]$. The significant factors are shown in Table 1. Judgments decreased with increasing absolute phase deviation. Phase $S D$ interacted with absolute phase deviation, yielding smaller coordination judgments especially when large phase variability occurred with small absolute phase deviations. Increasing frequency of motions also yielded smaller coordination judgments for motions with either large absolute phase deviation or large phase variability. Intended antiphase motions 
A
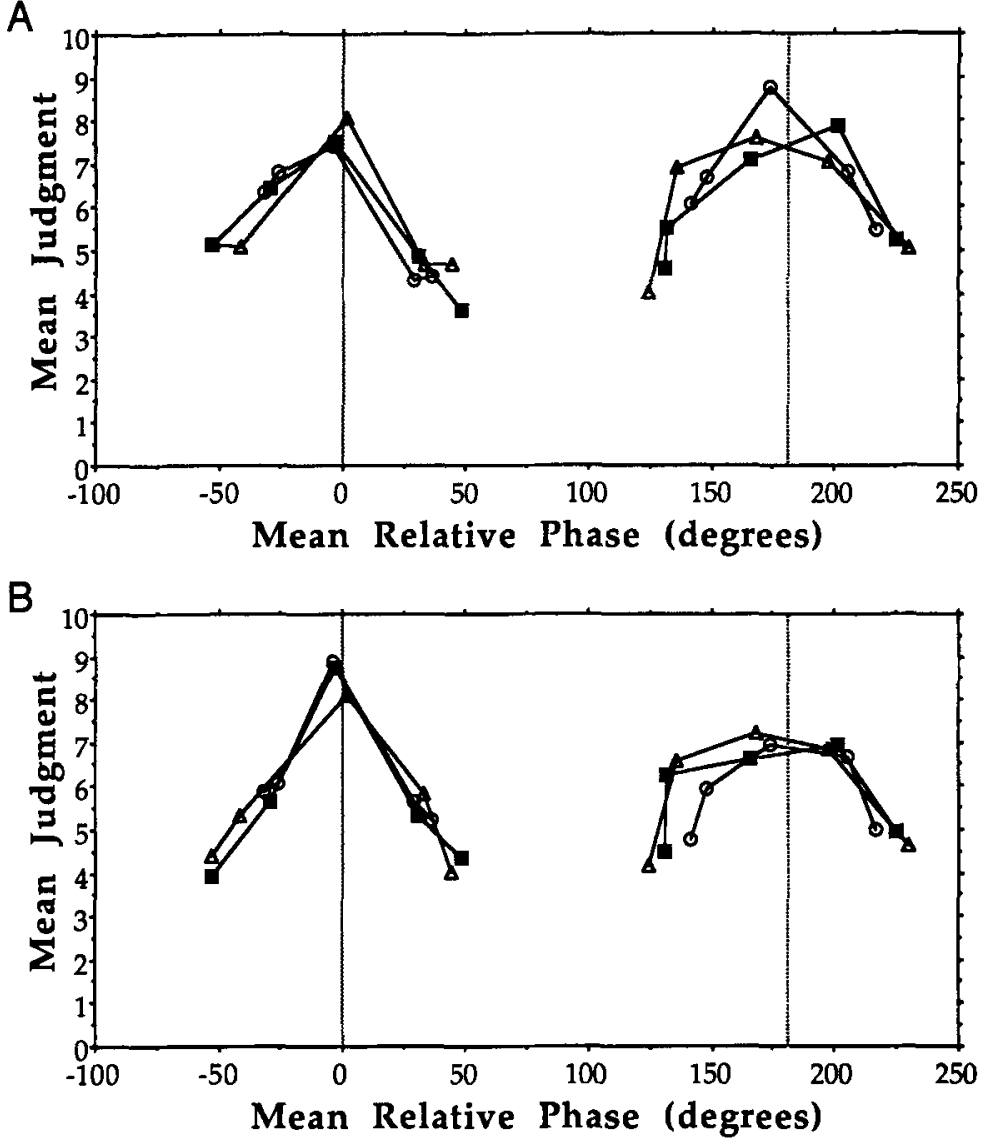

Figure 5. (A) Mean judgments in the side-on viewing condition of Experiment 1 plotted as a function of mean relative phase of the display. Means plotted separately for each frequency: $F 1=0.799 \mathrm{~Hz}$ (open circles); $F 2=0.936 \mathrm{~Hz}$ (filled squares); F3 $=1.151 \mathrm{~Hz}$ (open triangles). (B) Mean judgments in the in-depth viewing condition of Experiment 1 plotted as a function of mean relative phase of the display. Means plotted separately for each frequency: F1 $=0.799 \mathrm{~Hz}$ (open circles); F2 $=0.936 \mathrm{~Hz}$ (filled squares); F3 $=1.151 \mathrm{~Hz}$ (open triangles).

(i.e., motions with mean phases near $180^{\circ}$ ) were judged as less coordinated than intended in-phase motions (i.e., with mean phases near $0^{\circ}$ ). This occurred more with motions viewed in depth than with motions viewed side on.

Finally, we performed simple regressions, regressing either absolute phase deviation or phase $S D$ on mean judgments and on judgment $S D$ s separately for each participant. ${ }^{1}$ The mean $r^{2}$ and the percentage of the analyses that were significant at $p<.05$ or better are shown in Table 2 . The results confirmed the observation that judgments of coordination were primarily determined by the extent to which mean phases deviated from either $0^{\circ}$ or $180^{\circ}$. If either $0^{\circ}$ or $180^{\circ}$ were judged as maximally coordinated, then, by inference, $90^{\circ}$ or $270^{\circ}$ would be judged as least coordinated. Phase variability only seemed to affect judgments when it occurred with mean phases near $0^{\circ}$ or $180^{\circ}$. The results were essentially the same whether the motion was viewed side on or in depth.
A problem with Experiment 1 was that when we explained the task to the participants, we had only shown them motions at $0^{\circ}$ and $180^{\circ}$ mean phase as examples of fully coordinated motion. It was possible that they inferred from this demonstration that only those mean relative phases should be judged as coordinated. If so, then the results were an artifact produced by the instructions. We performed a control experiment to test this possibility.

We replicated Experiment 1 using the same displays with only viewing in depth. We changed the instructions in two ways to ensure that the participants understood that motions with mean phases other than $0^{\circ}$ or $180^{\circ}$ should be judged as coordinated if they exhibited low phase variability. First, we used circular diagrams to explain the difference between mean phase and phase variability. We showed how motions around two circles could be at the same mean phase (e.g., $90^{\circ}$ ) with different levels of phase variability (low or high) and how motions with the same 

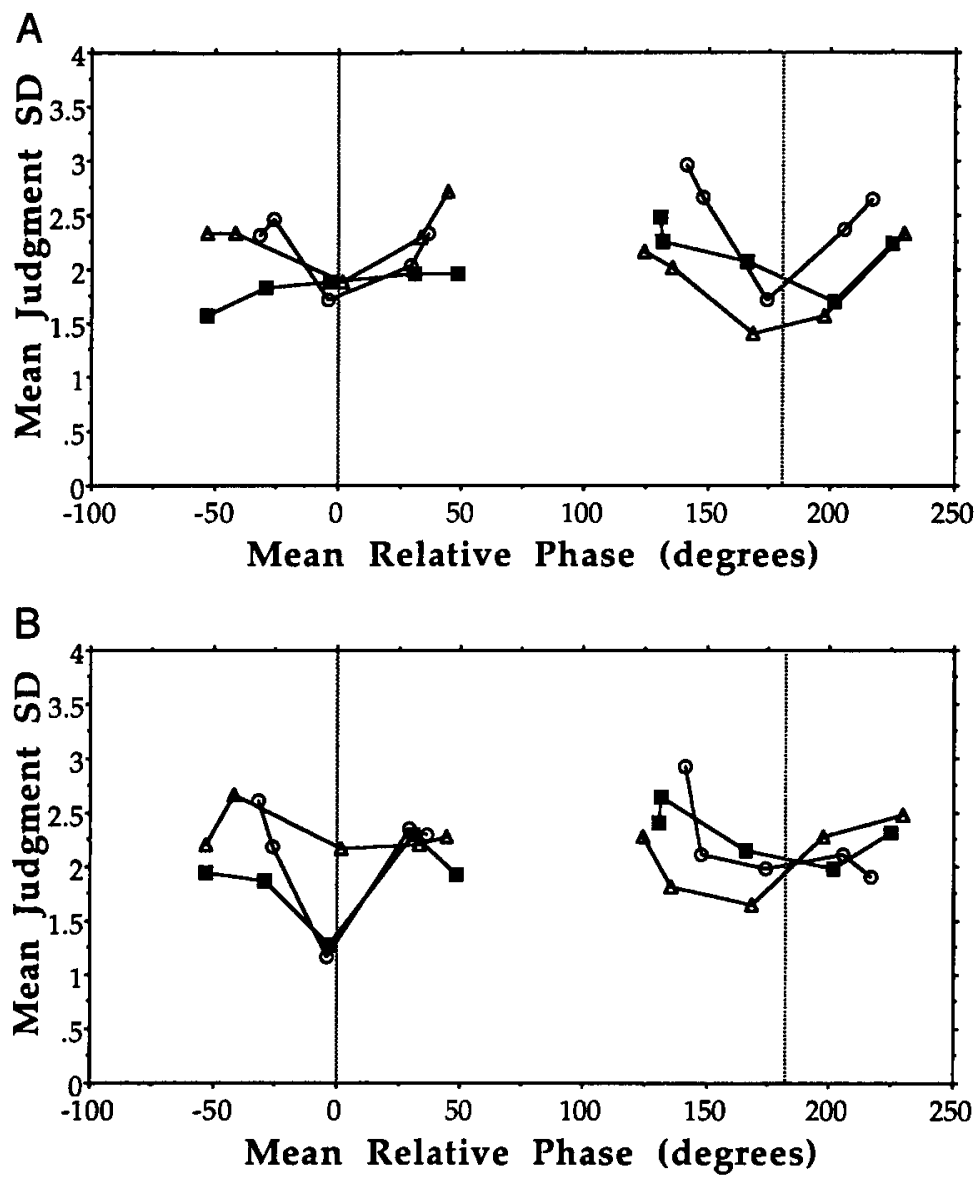

Figure 6. (A) Mean judgment standard deviations in the side-on viewing condition of Experiment 1 plotted as a function of mean relative phase of the display. Means plotted separately for each frequency: $F 1=0.799 \mathrm{~Hz}$ (open circles); $\mathrm{F} 2=$ $0.936 \mathrm{~Hz}$ (filled squares); F3 = $1.151 \mathrm{~Hz}$ (open triangles). (B) Mean judgment standard deviations in the in-depth viewing condition of Experiment 1 plotted as a function of mean relative phase of the display. Means plotted separately for each frequency: $\mathrm{F1}=0.799 \mathrm{~Hz}$ (open circles); $\mathrm{F2}=0.936 \mathrm{~Hz}$ (filled squares); F3 = $1.151 \mathrm{~Hz}$ (open triangles).

level of variability could be at different mean phases $\left(0^{\circ}\right.$ or $90^{\circ}$ ). We explained that judgments of coordination should vary with the amount of phase variability, but not with variations in mean phase. A motion at $90^{\circ}$ mean phase with low phase variability should be judged as highly co-

Table 1

Significant Factors Found in a Multiple Regression on Judgment Means Including the Data of Two Viewing Conditions, Side On and In Depth

\begin{tabular}{lccc}
\hline \multicolumn{1}{c}{ Factor } & \multicolumn{1}{c}{$p$} & $\beta$ & Partial $F$ \\
\hline PDev & $<.001$ & -.72 & 88.4 \\
PSD $\times$ PDev & $<.05$ & .18 & 4.6 \\
F $\times$ PDev & $<.03$ & -.36 & 5.2 \\
F $\times$ PSD $\times$ PDev & $<.01$ & .43 & 7.1 \\
IP & $<.01$ & .09 & 8.9 \\
$\mathrm{~V} \times \mathrm{IP}$ & $<.001$ & -.24 & 13.6 \\
$\mathrm{~V} \times \mathrm{IP} \times \mathrm{PDev}$ & $<.01$ & .19 & 8.2 \\
\hline
\end{tabular}

Note-PDev, absolute phase deviation; PSD, standard deviation of phase; $F$, frequency; IP, intended phase; $V$, viewing condition. ordinated. Second, among the demonstration displays illustrating highly coordinated motions, we included motions with relative phases of $0^{\circ}, 20^{\circ}, 40^{\circ}, 180^{\circ}, 200^{\circ}$, and $220^{\circ}$.

Eight undergraduates at Indiana University participated for introductory psychology course credit. None had participated in Experiment 1 and all had normal or correctedto-normal vision.

As before, we computed a mean and an SD of the coordination judgments for each cell and participant. We combined the data from the in-depth viewing condition of Experiment 1 with the control data and performed an ANOVA, with viewing condition as a between-subjects factor and with intended phase, frequency, and system as withinsubjects factors. Neither viewing nor any of the viewing interactions was significant. Otherwise, the results were the same as in Experiment 1, as shown in Figure 7A. When we performed the ANOVA on judgment $S D \mathrm{~s}$, neither viewing nor any of its interactions was significant, and the 
Table 2

Mean Results of Simple Regressions Performed on the Data of Each Participant in Three Viewing Conditions, Regressing Absolute Phase Deviations (PDev) or Standard Deviations of Phase (PSD) on Either Judgment Means or Standard Deviations (SDs)

\begin{tabular}{|c|c|c|c|c|c|c|c|c|c|}
\hline \multirow{3}{*}{$\begin{array}{l}\text { Viewing } \\
\text { Condition }\end{array}$} & \multirow[b]{3}{*}{$n$} & \multicolumn{4}{|c|}{ Judgment Means } & \multicolumn{4}{|c|}{ Judgment $S D s$} \\
\hline & & \multicolumn{2}{|c|}{ PDev } & \multicolumn{2}{|c|}{$\mathrm{PSD}$} & \multicolumn{2}{|c|}{$\mathrm{P} D e v$} & \multicolumn{2}{|c|}{$\mathrm{PSD}$} \\
\hline & & Mean $r^{2}$ & Percent* & Mean $r^{2}$ & Percent* & Mean $r^{2}$ & Percent* & Mean $r^{2}$ & Percent* \\
\hline Experiment 1 Side On & 13 & .35 & 100 & .05 & 0 & .12 & 31 & .02 & .8 \\
\hline Experiment 1 In Depth & 15 & .42 & 100 & .11 & 40 & .12 & 33 & .02 & 0 \\
\hline
\end{tabular}

Note $-n=$ number of subjects. $*$ Percent analyses $p<.05$ or better.

results were again the same as in Experiment 1, as shown in Figure 7B.

We performed a multiple regression of absolute phase, deviation, phase $S D$, frequency, intended phase, and instruction condition together with interaction vectors on the combined mean coordination judgments with in-depth viewing. After removing nonsignificant factors, the analysis accounted for $38 \%$ of the variance $[F(8,681)=51.6, p<$ $.001]$. The significant factors included both absolute phase deviation and phase variability, as shown in Table 3 . The factors other than absolute phase deviation, absolute phase deviation $\times$ phase $S D$ interaction, and instruction condition

A

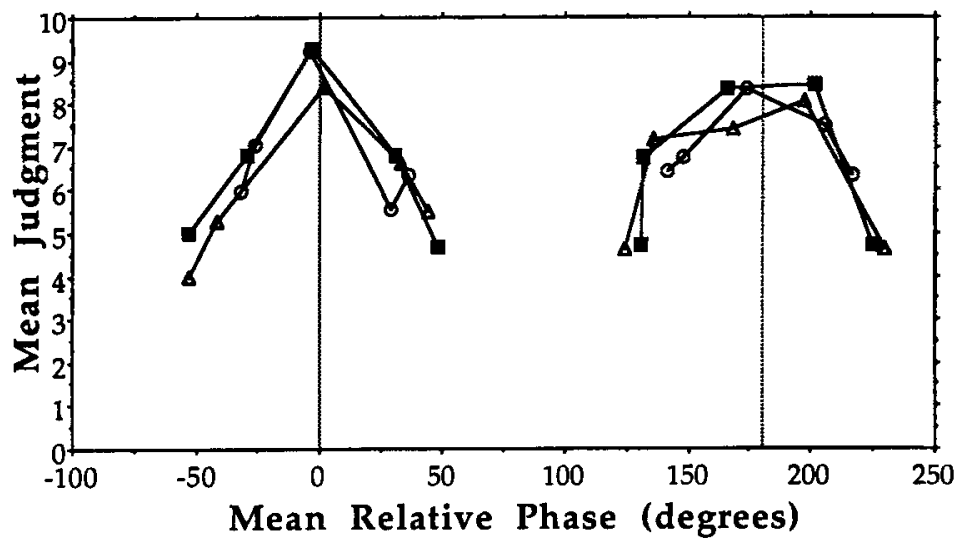

B

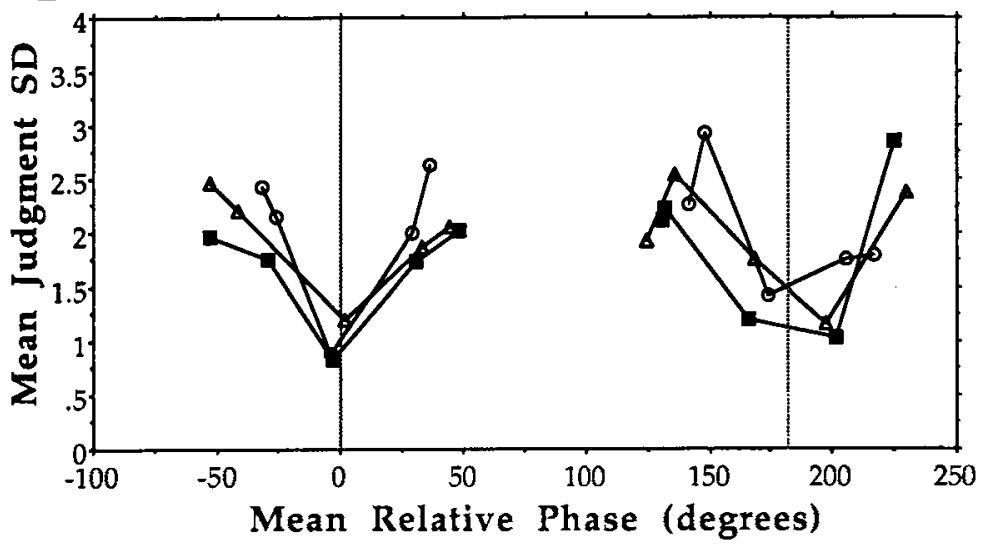

Figure 7. (A) Mean judgments of the control experiment plotted as a function of mean relative phase of the display. Means plotted separately for each frequency: $\mathrm{F1}=0.799 \mathrm{~Hz}$ (open circles); F2 $=0.936 \mathrm{~Hz}$ (filled squares); F3 $=1.151 \mathrm{~Hz}$ (open triangles). (B) Mean judgment standard deviations of the control experiment plotted as a function of mean relative phase of the display. Means plotted separately for each frequency: $F 1=0.799 \mathrm{~Hz}$ (open circles); $F 2=0.936 \mathrm{~Hz}$ (filled squares); $F 3=1.151 \mathrm{~Hz}$ (open triangles). 
Table 3

Significant Factors Found in a Multiple Regression on Judgment Means Including the In-Depth Viewing Data of Experiment 1 and the Control Experiment

\begin{tabular}{lccc}
\hline \multicolumn{1}{c}{ Factor } & \multicolumn{1}{c}{$p$} & \multicolumn{1}{c}{$\beta$} & Partial $F$ \\
\hline $\mathrm{P} D e v$ & $<.001$ & -1.1 & 79.0 \\
$\mathrm{PSD}$ & $<.001$ & -.29 & 12.5 \\
$\mathrm{PSD} \times \mathrm{P} D e v$ & $<.001$ & .70 & 18.0 \\
$\mathrm{IP} \times \mathrm{P}$ Dev & $<.05$ & -.08 & 4.6 \\
$\mathrm{~F} \times \mathrm{IP}$ & $<.01$ & .19 & 6.8 \\
$\mathrm{~F} \times \mathrm{IP} \times \mathrm{P}$ Dev & $<.001$ & -.66 & 12.1 \\
$\mathrm{~F} \times \mathrm{IP} \times \mathrm{PSD} \times \mathrm{P} D e v$ & $<.01$ & .52 & 9.7 \\
$\mathrm{I}$ & $<.001$ & -.16 & 26.3 \\
\hline
\end{tabular}

Note-PDev, absolute phase deviation; PSD, standard deviation of phase; IP, intended phase; F, frequency; I, instruction condition.

accounted for only $2 \%$ of the variance. The three factors alone yielded an $r^{2}$ of .36. The instructions in the control experiment yielded somewhat higher judgments overall.

In sum, we found that the instructions were not responsible for the results in Experiment 1. In general, coordination judgments covaried with absolute phase deviation with some effect of phase variability. Mean phases of $0^{\circ}$ and $180^{\circ}$ were judged most reliably and judged as most coordinated; $0^{\circ}$ was judged more reliably than $180^{\circ}$ and was judged as more coordinated than $180^{\circ}$. Greater phase variability yielded judgments of lower coordination.

\section{EXPERIMENT 2}

In Experiment 1, we used displays generated from recorded kinematics of bimanual movements. The advantage was that the results could be safely generalized to the visual perception of human movements and, by extension, to the results of studies of human coordination. However, the disadvantage of the method was that we could not independently manipulate mean phase and phase variability to sample the range of each systematically. The visual perception of relative phase as such might be better evaluated (beyond the context of human movement) without concurrent variations in the amplitude and frequency of movement. The goal of Experiment 2 was to replicate the previous results with precise control of both mean phase and phase variability. The participants were first tested in a blocked condition that exposed them to variations in phase variability blocked within levels of mean phase. This was intended to maximize and tune their ability to discriminate phase variability independent of mean phase. The participants were then tested in a design with a completely random ordering of displays.

\section{Method}

Participants. Ten undergraduates at Indiana University participated in the experiment. Four were men, and 6 were women. The participants ranged in age from 18 to 29 years. They were paid at $\$ 5$ per hour. None had participated in the previous studies. All had normal or corrected-to-normal vision.

Display generation. Displays consisted of two black rings $(2 \mathrm{~cm}$ in diameter) appearing on a white background. Each ring moved along a horizontal linear path $6 \mathrm{~cm}$ in length. The paths were centered horizontally on the screen. The paths were separated vertically on the screen by $5.5 \mathrm{~cm}$. Each ring moved with a simple harmonic motion at $1 \mathrm{~Hz}$. The two rings were programmed to oscillate at mean relative phases of $0^{\circ}, 30^{\circ}, 60^{\circ}, 90^{\circ}, 120^{\circ}, 150^{\circ}$, or $180^{\circ}$. Noise signals were added to the harmonic motions to produce specific levels of phase variability. Four levels of phase variability were produced at each of the seven mean phases. The $S D$ s of phase for the four levels were $0^{\circ}, 5^{\circ}, 10^{\circ}$, and $15^{\circ}$. This was accomplished as follows.

The frequency of the added noise signals was set equal to either $1,0.5$, or 0.25 times the frequency of the oscillators $(1 \mathrm{~Hz})$. The noise signals were used to alter the time used to create each harmonic motion. The temporal noise signal was

$$
N_{i}=A_{N_{i}} \cos \left(\omega_{N} t\right)+0.1 A_{N_{i}} \xi_{t},
$$

where $i=1,2$ specifies the oscillator, $A_{N}$ is the amplitude of the temporal noise, $\omega_{N}$ is the frequency of the noise, and $\xi_{t}$ is Gaussian white noise of unit variance. We constrained the temporal noise signal, $N_{i}$, to be smaller than a time step, that is, smaller than one $d t=$ $0.03 \mathrm{sec}$, so that the oscillator was slowed or speeded, but never stopped or reversed:

$$
N_{i}^{*}=\left[-.95<N_{i}<.95\right] .
$$

The temporal noise signal was used to determined the rate of increase of time:

$$
\frac{d t_{p_{i}}}{d t}=1+N_{i}^{*}
$$

Equation 1 was integrated numerically using a fourth order RungeKutta routine to yield approximately:

$$
t_{i}(n+1)=t_{i}(n)+\left[d t \times d t_{p_{i}}(n)\right],
$$

where $n$ is the number of the time step, $d t=0.03 \mathrm{sec}$, and $t_{i}(0)=0$. This determined the phase of an oscillator as follows:

$$
\phi_{i=} \omega_{H} t_{i}+\Delta \phi_{i}
$$

where $\omega_{H}$ is the oscillator frequency, and $\Delta \phi_{i}$ is the mean phase of the $i$ th oscillator. Finally, the motion of each oscillator was generated as

$$
X_{i}(t)=A_{p_{i}} \cos \left(\phi_{i}\right)
$$

where $A_{p}$ is the amplitude of the oscillators (i.e., $3 \mathrm{~cm}$ on the screen). The noise was added to the oscillators in three different ways to produce each level of phase variability. First, noise signals of equal amplitude and opposite phase were added to each oscillator. Second, noise signals with one amplitude triple the other were added with equivalent phase. Third, a noise signal was added to only one of the oscillators. A constrained random procedure was used to determine which oscillator received the (larger) perturbation in the (second) third method so that each received it equally often.

Seven mean phases $\times 4$ levels of phase variability $\times 3$ ways of adding phase variability yielded 84 displays. Displays were generated with a frame rate of $33 \mathrm{~Hz}$. They were viewed using normal binocular vision in a dimly lit room at a distance of $0.7 \mathrm{~m}$ from the screen. Head motion and eye motion were unconstrained. A response bar appeared after each display. The horizontal bar was numbered from 0 to 10 and extended beyond both 0 and 10 to avoid artifactual reduction of response variability at 0 and 10 due to the end stops. A marker could be moved continuously along the bar and freely adjusted using the mouse. Double clicking the mouse both entered the judgment value and started the next trial.

Procedure. The participants were instructed to judge phase variability. The difference between mean phase and phase variability was explained to them. The participants were then shown a number of demonstrations of different mean phases and different levels of phase variability. The use of the response bar was also illustrated, 


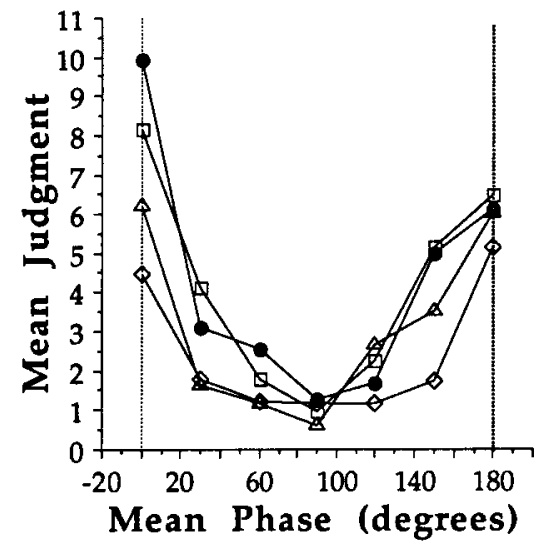

Figure 8. Mean judgments of Experiment 2 plotted as a function of mean relative phase of the display. Means plotted separately for each level of phase variability: Phase $S D=0^{\circ}$ (filled circles); Phase $S D=5^{\circ}$ (open squares); Phase $S D=10^{\circ}$ (open triangles); Phase $S D=15^{\circ}$ (open diamonds).

and the participants were allowed to practice with it on the demonstration displays. The participants then performed the judgment task in a blocked condition. Trials were blocked by mean relative phase in increasing order from $0^{\circ}$ to $180^{\circ}$ - that is, they judged random order presentations of the four different levels of phase variability (12 trials), all presented with $0^{\circ}$ mean relative phase. Next, they judged phase variability for motions at $30^{\circ}$ mean phase, and so on. After the blocked session was completed, the participants judged the entire set of displays in a completely random order.

\section{Results}

We computed a mean and an $S D$ of the coordination judgments for each cell and participant. We report only the results from the session with fully randomized displays. We performed a repeated measures ANOVA on the means, with phase variability (4 levels) and mean phase ( 7 levels) as factors. Phase variability was significant $[F(3,27)$ $=21.7, p<.001]$. In Tukey post hoc tests, judgments of phase $S D 0^{\circ}$ and $5^{\circ}$ were different from those of $S D 10^{\circ}$ and $15^{\circ}$. The participants were able to distinguish different levels of phase variability. Mean phase was significant $[F(6,54)=8.3, p<.001]$. Judgments of mean phases of $0^{\circ}$ and $180^{\circ}$ were different from judgments of mean phases of $60^{\circ}, 90^{\circ}$, and $120^{\circ}$. Finally, the phase variability $\times$ mean phase interaction was significant $[F(18,162)=3.0$, $p<.001]$. In simple effects tests, phase variability was significant (or marginal) at mean phases of $0^{\circ}(p<.001)$, $30^{\circ}(p<.04), 150^{\circ}(p<.01)$, and $180^{\circ}(p<.06)$. Mean phase was significant at phase $S D$ s of $0^{\circ}, 5^{\circ}$, and $10^{\circ}(p<$ $.001)$ and at $S D 15^{\circ}(p<.03)$. Thus, as shown in Figure 8, the participants were able to discriminate levels of phase variability when mean phase was at or near $0^{\circ}$ or $180^{\circ}$, but not when mean phase was at or near $90^{\circ}$. Furthermore, as mean phase departed from $0^{\circ}$ or $180^{\circ}$, the motion was judged as having greater phase variability, and this was true especially when there was no phase variability at all.

We performed the same ANOVA on the judgment $S D$ s. Phase variability was significant $[F(3,27)=3.2, p<.04]$, and post hoc $t$ tests revealed that phase $S D$ s of $0^{\circ}$ and $5^{\circ}$ were each different from $S D 10^{\circ}$. Mean phase was significant $[F(6,54)=6.1, p<.001]$. The phase variability $\times$ mean phase interaction was not significant $(p=.2)$. In simple effects tests, phase variability was significant at $0^{\circ}$ mean phase only where judgment variability covaried with phase variability. Mean phase was significant at phase $S D$ s of $0^{\circ}$ and $5^{\circ}(p<.001)$ and at $S D 10^{\circ}(p<.05)$, but not at $S D 15^{\circ}$. Judgment variability increased as mean phase departed from either $0^{\circ}$ or $180^{\circ}$, peaking at $90^{\circ}$; however, judgment variability was already high as a function of high phase variability and so was not affected by mean phase.

These results confirmed those obtained in Experiment 1 , showing that judgments of phase variability (or how coordinated movements are) are affected by both the actual phase variability and the mean phase. At $0^{\circ}$ and $180^{\circ}$ mean phase, different levels of phase variability are discriminated; however, at $90^{\circ}$ mean phase, they are not. With no phase variability, mean phases other than $0^{\circ}$ and $180^{\circ}$ are judged as more variable or less coordinated. To make clear this latter pattern, we performed repeated measures ANOVAs on the means and $S D$ s of judgments of $0^{\circ}$ phase $S D$ movements (i.e., movements varying only in mean relative phase with no phase variability). Mean phase was, of course, the only factor. Both analyses were significant [judgment means $F(6,54)=11.3, p<.001$; judgment $S D$ s, $F(6,54)=4.2, p<.005]$. Post hoc $t$ tests revealed that mean judgments of $0^{\circ}$ mean phase were different $(p<.01)$ from all other mean phases and, in particular, from judgments of $180^{\circ}$ mean phase that were judged to be more variable than $0^{\circ}$ mean phase movements (despite the absence of phase variability). Similarly, post hoc $t$ tests of judgment $S D$ s revealed that judgments of $0^{\circ}$ mean phase were less variable $(p<.05)$ than at all other mean phases including $180^{\circ}$. This last analysis reveals that movements at $180^{\circ}$ mean relative phase are judged to be less stable with respect to relative phase than are movements at $0^{\circ}$ mean relative phase.

\section{DISCUSSION}

In the HKB model of interlimb coordination, relative phase $(\phi)$ is characterized as an order parameter, whereas frequency of oscillation is called a control parameter. The implication is that phase is not directly controlled and that only frequency is controlled via the stiffness of a massspring control dynamic. However, a number of circumstances imply, to the contrary, that relative phase is both a controlled property and a perceptible property. We assume that, if a property or variable is to be controlled, then it must be perceptible. (Of course, the reverse need not be true.)

The first circumstance is that participants in the coordination experiments are explicitly instructed to oscillate two limbs either at an in-phase or an antiphase relation. Participants are able to do this reliably, although the relative phase actually produced can be perturbed away from 
$0^{\circ}$ or $180^{\circ}$ by manipulating the inherent frequencies (inertias) of the two oscillators. The fact that people are able to reliably distinguish and generate two different relative phases means that they are able to control their behavior with respect to this property, and, thus, they must be able to perceive it. However, the fact that people without special training or skill cannot reliably produce arbitrary relative phases (Zanone \& Kelso, 1992) or that phase deviations produced by inertial asymmetry are not typically detected implies that the ability to resolve relative phase varies depending on relative phase.

The second circumstance is that the bimanual coordination results were reproduced in experiments in which coordination was maintained between 2 people who used vision to establish and maintain a given phase relation (Schmidt et al., 1990; Schmidt \& Turvey, 1994). This implies that relative phase is a visually perceptible property.

The third circumstance is perhaps the most significant. Kelso, Scholz, and Schoner (1986) have reported a number of experiments in which, as the frequency of oscillation has been increased, participants have switched from a $180^{\circ}$ relative phase to a $0^{\circ}$ relation, putatively because the $180^{\circ}$ phase has become unstable and too difficult to maintain at higher frequencies of oscillation. Switching does not occur when participants start the frequency series at $0^{\circ}$ relative phase. They maintain the initial relative phase throughout a given trial. Less well known is the fact that not all participants always exhibit this switching. For instance, Zelaznik, Smith, Franz, and Ho (1997) found that transitions occurred in only a small proportion of trials with finger oscillations, and transitions have not occurred in experiments involving wrist pendulums (Schmidt, personal communication, August, 1997). Lee, Blandin, and Proteau (1996) also found resistance to switching in a bimanual task. The original experiments entailed a noninterference paradigm akin to that used by Feldman in establishing the $\lambda$ model of limb movement: Participants were instructed not to resist when they felt an inclination to switch. The problem is that a participant must evaluate an inclination. Some participants can go through the entire frequency series in the antiphase mode without switching. Of course, the phase variability of oscillation increases with increasing frequency, but the mean antiphase relation can sometimes be maintained nevertheless. The problem is to decide, as the fluctuations become increasingly large and more frequent, when to stop correcting in response to fluctuations and to switch. It is possible to select a given level of difficulty and consistently allow oneself to switch at that point. Ultimately, all participants must do this in cooperating with the "noninterference" instructions. The point is that they must be able to perceive the level of difficulty determined by the amount of phase variability. This implies, in turn, the perceptibility of phase variability. This realization inspired the present experiments. The fact that the switching behavior was reproduced in the between-people coordination experiments implied that phase variability could also be perceived visually. Summers, Thomas, and Byblow (1997) studied spontaneous switching versus intentional switching upon a command. The authors observed what they called intentional corrections; however, they had no clear criteria for reliably identifying all such corrections. Without such criteria, there could be no assurance that spontaneous switches were not intentional, in the sense that corrections were intentionally curtailed.

In the present experiments, we tested the ability to perceive relative phase variability in human movements or relative phase stability. The somewhat surprising outcome was that judgments of phase stability were better predicted by mean absolute relative phase deviations from $0^{\circ}$ or $180^{\circ}$ relative phase, although relative phase variability also predicted judgments when mean relative phases were at $0^{\circ}$ or $180^{\circ}$. This was true of both mean judgments and judgment $S D$ s. However, using human movements to generate our displays prevented us from controlling mean relative phase and phase variability entirely independently. So, we resorted to simulations to produce oscillations at selected mean relative phases from $0^{\circ}$ to $180^{\circ}$, each with levels of phase variability ranging from $0^{\circ}$ to $15^{\circ}$ phase $S D$ (i.e., from no phase variability to modest amounts). In this case, the participants were given extensive instructions and demonstrations and were tested in a preliminary training session in which trials were blocked by mean phase to ensure that they understood the task. The results replicated our findings with human movement displays. Overall, judgments of the amount of relative phase variability were best predicted by mean relative phase. For instance, with no relative phase variability in the displayed movement, both judgment means and $S D$ s varied with the mean relative phase of the movement; $0^{\circ}$ and $180^{\circ}$ phase were judged as least variable and were judged so most reliably. Furthermore, $0^{\circ}$ was judged as less variable and more reliably so than $180^{\circ}$, whereas $90^{\circ}$ relative phase was judged as most variable but was judged so least reliably. When relative phase variability was added to the movements, it did not affect the judgments of movements at $90^{\circ}$ mean relative phase, but it did affect judgments of movements at $0^{\circ}$ and $180^{\circ}$. The latter were judged as more variable and were judged so less reliably.

In short, mean relative phase and the variability of relative phase interacted in determining judgments of relative phase stability. Movements at mean phases other than $0^{\circ}$ and $180^{\circ}$ and, especially, movements at $90^{\circ}$ were seen as more variable, and the participants could not distinguish relative phase variability from movements at these mean relative phases. Movement at $90^{\circ}$ simply appears variable, whereas $180^{\circ}$ is inherently perceived as more variable than $0^{\circ}$. This pattern of results is comparable to that of the bimanual coordination experiments.

Mean relative phase relations that are confused with relative phase variability or perceived as inherently variable would be difficult to control. In fact, such relative phases are also judged unreliably. In the coordination experiments, a stable phase relation should correspond to a relative phase that is readily and thus reliably distinguished from neighboring relative phases and from rela- 

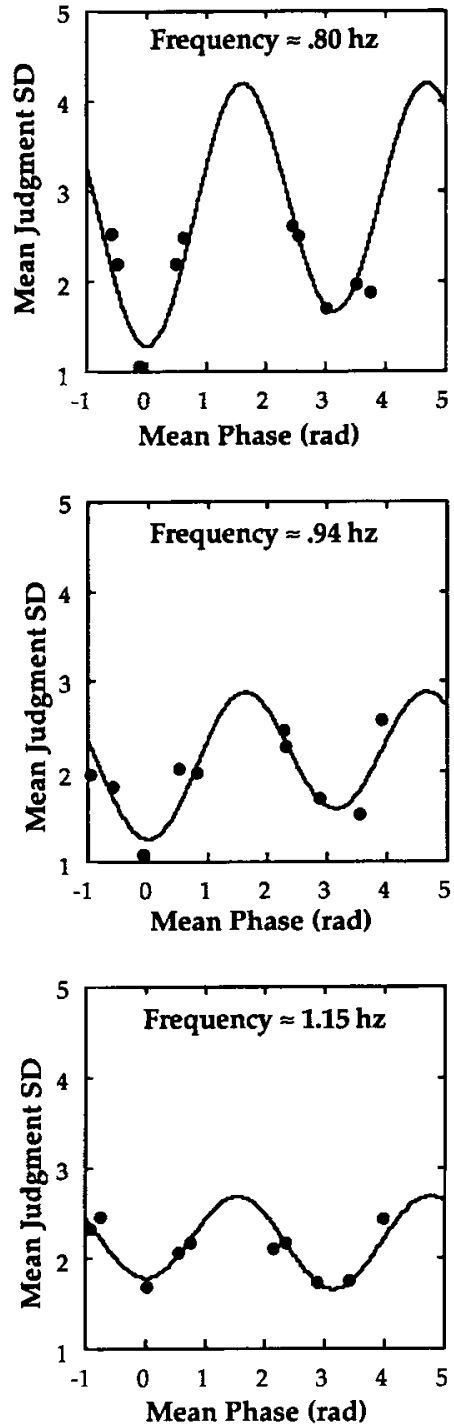

Figure 9. The HKB model fitted to the mean judgment standard deviations from the in-depth viewing condition at each of the three frequencies. See the text for additional explanation and compare with Figure 1.

tive phase variability when it occurs. Relative phases that are difficult to discriminate should be unstable and difficult to maintain. The potential function described in the $\mathrm{HKB}$ model is meant to represent the relative difficulty or effort required to maintain a given relative phase. The relative phase that corresponds to the bottom of a potential well is maintained with less difficulty than the neighboring phases and, thus, we infer, should also be discriminated from them more reliably.

To compare the HKB model with our judgments, we fitted the model to the mean $S D$ s of the judgments from the in-depth viewing condition. We added a parameter $(c)$ to the model to enable it to adjust to the absolute sizes of the $S D$ s: $V(\phi)=-a \cos (\phi)-b \cos (2 \phi)+c$. We fitted the model separately to data from each of the three frequency conditions. The fits were accomplished using the quasinewton method of estimation in SYSTAT (Wilkinson, Hill, \& Vang, 1992). Assuming that the model would fit the data fairly well, the questions were (1) whether the depth of the potential well at $0^{\circ}$ would be greater than that at $180^{\circ}$ (2) whether the depth of the potential wells would decrease with increases in frequency, and (3) if so, whether this would occur more at $180^{\circ}$ than at $0^{\circ}$. The results are shown in Figure 9, where it is apparent that the fit of the model was quite good. The $r^{2} \mathrm{~s}$ were $.982, .987$, and .996 for the low frequency, medium frequency, and high frequency, respectively. ${ }^{2}$ The potential well at $0^{\circ}$ was deeper than that at $180^{\circ}$ at the first two frequencies, but not at the third. The potential wells became more shallow with increasing frequency, but they did so equally at $0^{\circ}$ and $180^{\circ}$, a bit more so at $0^{\circ}$. When we fit the function to the mean judgment $S D$ s of Experiment 2 (i.e., the simulation), the result was similar; however, there was only a single frequency of movement, and a stronger asymmetry was obtained between $0^{\circ}$ and $180^{\circ}$ with the well at $0^{\circ}$ considerably deeper.

Thus, the fit of the model was quite good, and the results conformed to those of the coordination experiments in all but one respect - namely, the relative change in the depth of the wells at $0^{\circ}$ and $180^{\circ}$. However, there are two qualifying conditions. First, the frequencies in this case did not approach those where switching has been observed in the finger oscillation experiments, and the oscillations observed in this experiment were produced by wrist-pendulum systems that have not reproduced the switching behavior. Second, and most important, because these displays were generated by human movements, the frequency variations were accompanied by increases in phase variability even at $0^{\circ}$ and $180^{\circ}$ mean phase. To establish the effect of increasing frequency on the perception of relative phase, we must perform a test in which frequency increases are not accompanied by changes in phase variability. So, evaluation of this last aspect must await further study. Shy of this, we have found evidence supporting the possibility that the results of the studies on bimanual coordination are a product of perceptual abilitynamely, the ability to discriminate specific mean relative phases from phase variability. The conclusion is that relative phase is a perceptible property and that this perceptual ability merits further study.

\section{REFERENCES}

Bertenthal, B. I., \& Pinto, J. (1993). Complementary processes in the perception and production of human movements. In L. B. Smith \& E. Thelen (Eds.). A dynamic systems approach to development: $A p-$ plications (pp. 209-239). Cambridge, MA: MIT Press

Bingham, G. P., Schmidt, R. C.. Turvey, M. T., \& Rosenblum, L. D. (1991). Task dynamics and resource dynamics in the assembly of a coordinated rhythmic activity. Journal of Experimental Psychology: Human Perception \& Performance, 17, 359-381.

Bingham, G. P., Schmidt, R. C., \& ZaAL, F. (1996, November). Visual perception of relative phase. Paper presented at the annual meeting of the Psychonomic Society, Chicago.

Fuchs, A., \& Kelso. J. A. S. (1994). A theoretical note on models of in- 
terlimb coordination. Journal of Experimental Psychology: Human Perception \& Performance, 20, 1088-1097.

HaKeN, H., Kelso, J. A. S., \& Bunz, H. (1985). A theoretical model of phase transitions in human hand movements. Biological Cybernetics, 51, 347-356.

Johansson, G. (1994). Configurations in event perception. In G. Jansson, S. S. Bergstrom \& W. Epstein (Eds.) Perceiving events and objects (pp. 29-122). Hillsdale, NJ: Erlbaum. (Original work published 1950)

Kelso, J. A. S. (1984). Phase transitions and critical behavior in human bimanual coordination. American Journal of Physiology: Regulatory, Integrative \& Comparative Phvsiologv, 15, R1000-R1004.

KeLSo, J. A. S. (1995). Dynamic patterns: The self-organization of brain and behavior. Cambridge, MA: MIT Press.

KELSO, J. A. S., \& JEKA, J. J. (1992). Symmetry breaking dynamics of human multilimb coordination. Journal of Experimental Psychology: Human Perception \& Performance, 18, 645-668.

Kelso, J. A. S., Scholz, J. P., \& Schoner, G. (1986). Non-equilibrium phase transitions in coordinated biological motion: Critical fluctuations. Physics Letters A, 118, 279-284.

Kelso, J. A. S., Schoner, G., Scholz, J. P., \& Haken, H. (1987). Phase locked modes, phase transitions and the component oscillators in biological motions. Phvsica Scripta, 35, 79-87.

Lee, T. D., Blandin, Y., \& Proteau, L. (1996). Effects of task instructions and oscillation frequency on bimanual coordination. Psychological Research, 59, 100-106.

NALWA, V. S. (1993). A guided tour of computer vision. Reading, MA: Addison-Wesley.

Pedhazur, E. (1982). Multiple regression in behavioral analysis. New York: Holt, Rinehart \& Winston.

Rosenblum, L. D., \& Turvey, M. T. (1988). Maintenance tendency in coordinated rhythmic movements: Relative fluctuations and phase. Neuroscience, 27, 289-300.

Schmidt, R. C., Beek, P. J., Treffner, P., \& Turvey, M. T. (1991), Dynamical substructure of coordinated rhythmic movements. Journal of Experimental Psychology: Human Perception \& Performance, 17, 635-651.

Schmidt, R. C., Carello, C., \& Turvey, M. T. (1990). Phase transitions and critical fluctuations in the visual coordination of rhythmic movements between people. Journal of Experimental Psychology: Human Perception \& Performance, 16, 227-247.

SChmidT, R. C., Shaw, B. K., \& Turvey, M. T. (1993). Coupling dynamics in interlimb coordination. Journal of Experimental Psychology: Human Perception \& Performance, 19, 397-415.

SCHMIDT, R. C., \& TURveY, M. T. (1994). Phase-entrainment dynamics of visually coupled rhythmic movements. Biological Cybernetics, 70. 369-376.

Schmidt, R. C., \& Turvey, M. T. (1995). Models of interlimb coordination: Equilibria, local analyses, and spectral patterning. Journal of Experimental Psychology: Human Perception \& Performance, 21, $432-443$

SCHOlZ, J. P., Kelso, J. A. S., \& Schoner, G. (1987). Non-equilibrium phase transitions in coordinated biological motion: Critical slowing down and switching time. Physics Letters A, 123, 390-394.
Schoner, G., \& Kelso, J. A. S. (1988). Dynamic pattern generation in behavioral and neural systems. Science, 239, 1513-1520.

Sternad, D., Turvey, M. T., \& Schmidt, R. C. (1992). Average phase difference theory and $1: 1$ phase entrainment in interlimb coordination. Biological Cybernetics, 67, 223-231.

Summers, J., Thomas, J., \& B yblow, W. (1997, July). Spontaneous and intentional movement transitions in Parkinson's disease. Paper presented at the 9th International Conference on Perception and Action, University of Toronto.

Tuller, B., \& Kelso, J. A. S. (1989). Environmentally-specified patterns of movement coordination in normal and split-brain subjects. Experimental Brain Research, 75, 306-316.

Turvey, M. T., Rosenblum, L. D., Schmidt, R. C., \& Kugler, P. N. (1986). Fluctuations and phase symmetry in coordinated rhythmic movements. Journal of Experimental Psychology: Human Perception \& Performance, $12,564-583$

Wilkinson, L., HILl, M. A., \& VANG, E. (1992). SYSTAT: Statistics, Version 5.2 [Computer software and manual]. Evanston, IL: SYSTAT.

Yaminishi, J., Kawato, M., \& Suzuki, R. (1979). Studies on human finger tapping neural networks by phase transition curves. Biological Cybernetics, 33, 199-208.

Yaminishi, J., KaWato, M., \& SuZUKi, R. (1980). Two coupled oscillators as a model for the coordinated finger tapping by both hands. Biological Cybernetics, 37, 219-225.

ZaAl, F., BinghaM, G. P., \& Schmidt, R. C. (1996, November). Visual perception of phase variability and mean phase. Paper presented at the annual meeting of the Psychonomic Society, Chicago.

ZANONE, P. G., \& KELSO, J. A. S. (1992). The evolution of behavioral attractors with learning: Nonequilibrium phase transitions. Journal of Experimental Psychology: Human Perception \& Performance, 18, 403-421.

Zelaznik, H. N., Smith, A., Franz, E. A., \& Ho, M. (1997). Differences in bimanual coordination associated with stuttering. Acta $P_{S y-}$ chologica, 96, 229-243.

\section{NOTES}

1. There were 4 trials in each cell for each observer. These included 2 repetitions of trials from each of 2 participants from the original Schmidt et al. (1993). We computed the $S D$ in mean phase for the trials from the 2 participants in each cell and regressed the set of these on the judgment $S D$ s for each observer. The mean $r^{2}$ and percentage of analyses significant at $p<.05$ or better were as follows: Experiment 1 side on, $.05 / 21 \%$; Experiment 1 in depth, $.06 / 13 \%$. In a multiple regression in which these within-cell $S D$ s in mean phase were combined with absolute phase deviations, the result was that the within-cell $S D$ s uniquely accounted for about $2 \%$ of the variance

2 . We regressed the within-cell $S D$ s in mean phase on judgment $S D$ s and derived residual scores. We then computed means of these residuals and fitted the model to these means for each frequency with comparable results. The $r^{2}$ s were $.987, .986$, and .992 , respectively.

(Manuscript received August 12, 1997; revision accepted for publication February 4, 1998.) 